\title{
The Logistic Lomax Distribution with Properties and Applications
}

\author{
Arun Kumar Chaudhary, Vijay Kumar
}

\begin{abstract}
A continuous univariate distribution called Logistic Lomax distribution is presented and studied. Relevant properties such as quantile function, survival function, and cumulative distribution function (CDF), probability density function (PDF), kurtosis and skewness measures are presented. For the parameter estimates of the presented distribution, Maximum likelihood Estimation (MLE) is used along with Cramer-Von-Mises estimation (CVME) and least-square estimation (LSE) methods. With the help of a real data set, we evaluate the goodness of fit of the distribution in comparison with other established distributions.
\end{abstract}

Index Terms- Quantile function, Logistic Lomax distribution, Reliability function, MLE

\section{INTRODUCTION}

Lifetime distributions are generally used to study the length of the life of components of a system. Lifetime distributions are frequently used in fields like life science, biology, engineering, insurance, etc. Many continuous probability distributions such as Cauchy, exponential, gamma, Weibull have been frequently used in statistical literature for analyzing lifetime data. For a few years, most of the researchers are attracted towards one parameter Logistic distribution for its potential in modeling lifetime data with exceptional performance in various applications

The logistic distribution is used for modeling logit models, logistic regression, growth models and neural networks. Though the logistic and normal distribution are very close in shape but we observe that in contrast to normal distribution, logistic distribution has slightly longer tails therefore providing more consistency with the underlying data. We can see the use of this model in finance, sports modeling and physical sciences.

Let $X$ denote non negative variable having shape parameter $\theta$ $>0$ and following the logistic distribution then its CDF can be expressed as,

$$
G(x ; \theta)=\frac{1}{1+e^{-\theta x}} ; \quad \theta>0, x \in \mathfrak{R}
$$

and its PDF is

$$
g(x ; \theta)=\frac{\theta e^{-\theta x}}{\left(1+e^{-\theta x}\right)^{2}} ; \quad \theta>0, x \in \mathfrak{R}
$$

Tahir et al. (2016) has introduced new family of continuous distributions obtained from a logistic random variable named as logistic-X family with varying properties.

Arun Kumar Chaudhary, Department of Management Science (Statistics), Nepal Commerce Campus, Kathmandu, Nepal

Vijay Kumar, Department of Mathematics and Statistics, DDU Gorakhpur University, Gorakhpur, India
Joshi et al. (2020) has introduced Logistic exponential power containing three parameters. For survival analysis, in comparison to modified Weibull distribution, a more flexibile distribution called Logistic-modified Weibull distribution was presented by Mandouh (2018). Joshi and Kumar (2020) have created half-logistic NHE distribution and studied its various mathematical and statistical properties. Chaudhary \& Kumar (2020) have presented the half- logistic exponential extension distribution using the parent distribution as exponential extension distribution. . Chaudhary \& Kumar (2020) also introduced Lindley half Cauchy distribution describing its relevant distributional properties. Kumar (2010) have presented the Bayesian analysis of exponential extension distribution.

A method to describe logistic compounded model was given by Lan and Leemis (2008) where they also proposed the logistic-exponential survival distribution with the help of same method. For lifetime modeling, this distribution has some useful properties such as bathtub and upside bathtub classes which demonstrates closed-form density. The logistic-exponential distribution's survival function can be expressed

$$
S(x ; \lambda)=\frac{1}{1+\left(e^{\lambda x}-1\right)^{\alpha}} ; \quad \alpha>0, \lambda>0, x \geq 0
$$

Using the same method as given by (Lan \& Leemis, 2008), Logistic Lomax distribution has been presented. In this study we have taken heavy-tailed shaped distribution called Lomax distribution as parent distribution which was presented by Lomax (1954). Inserting one extra parameter to Lomax distribution, we aim to introduce a distribution with more flexibility for obtain a better fit while modeling the lifetime dataset. The Lomax or Pareto Type II distribution is used in areas like life science, medicine, engineering, and many more. It can also be used in reliability and life testing problems in engineering and in reliability analysis as an alternative distribution (Hassan \& Al-Ghamdi, 2009). The CDF and PDF of Lomax distribution respectively can be written as

$$
F(x, \alpha, \beta)=1+(1+\beta x)^{-\alpha} ;(\alpha, \beta)>0
$$

and

$$
f(x, \alpha, \beta)=\alpha \beta(1+\beta x)^{-(\alpha+1)} ;(\alpha, \beta)>0
$$

The article shows following structure. In Section 2 we present the Poisson Gompertz distribution with discussion of some relevant distributional properties. For the parameter estimates of the presented distribution, MLE estimates is used along with CVME and LSE methods in Section 3. With the help of a real data set we evaluate the goodness of fit of the distribution in comparison with other established distribution 
in Section 4. We give the conclusion in Section 5.

\section{LOGISTIC LOMAX (LL) DISTRIBUTION}

In this section, we have introduced logistic Lomax (LL) distribution. In this study, we have taken the Lomax as baseline distribution. Consider $X$ denote non- negative random variable having a positive scale parameter $\beta$ and positive shape parameters $\alpha$ and $\lambda$, then logistic Lomax distribution's CDF is given as,

$$
F(x)=1-\frac{1}{1+\left((1+x \beta)^{\lambda}-1\right)^{\alpha}}
$$

; $x \geq 0, \alpha>0, \beta>0, \lambda>0$.

The PDF of LL distribution is

$$
f(x)=\frac{\lambda \beta \alpha(\beta x+1)^{\lambda-1}\left((\beta x+1)^{\lambda}-1\right)^{\alpha-1}}{\left\{1+\left((\beta x+1)^{\lambda}-1\right)^{\alpha}\right\}^{2}}
$$

$; x \geq 0$.

In comparison of CDF of logistic lomax to log logistic CDF function, we see second term of the denominator being changed in its base to Lomax function thus we termed the distribution to logistic Lomax distribution.

The reliability function of LL distribution

$$
\text { is } R(x)=1-F(x)=\frac{1}{1+\left\{(1+\beta x)^{\lambda}-1\right\}^{\alpha}}
$$

The failure rate function of LL distribution can be defined as,

$h(x)=\frac{f(x)}{R(x)}=\frac{\alpha \beta \lambda(1+\beta x)^{\lambda-1}\left\{(1+\beta x)^{\lambda}-1\right\}^{\alpha-1}}{1+\left\{(1+\beta x)^{\lambda}-1\right\}^{\alpha}}$

$; x \geq 0,(\alpha, \beta, \lambda)>0$

For different values of $\alpha, \beta$ and $\lambda$, in Figure 1, we have plotted PDF and hazard rate function of LL distribution
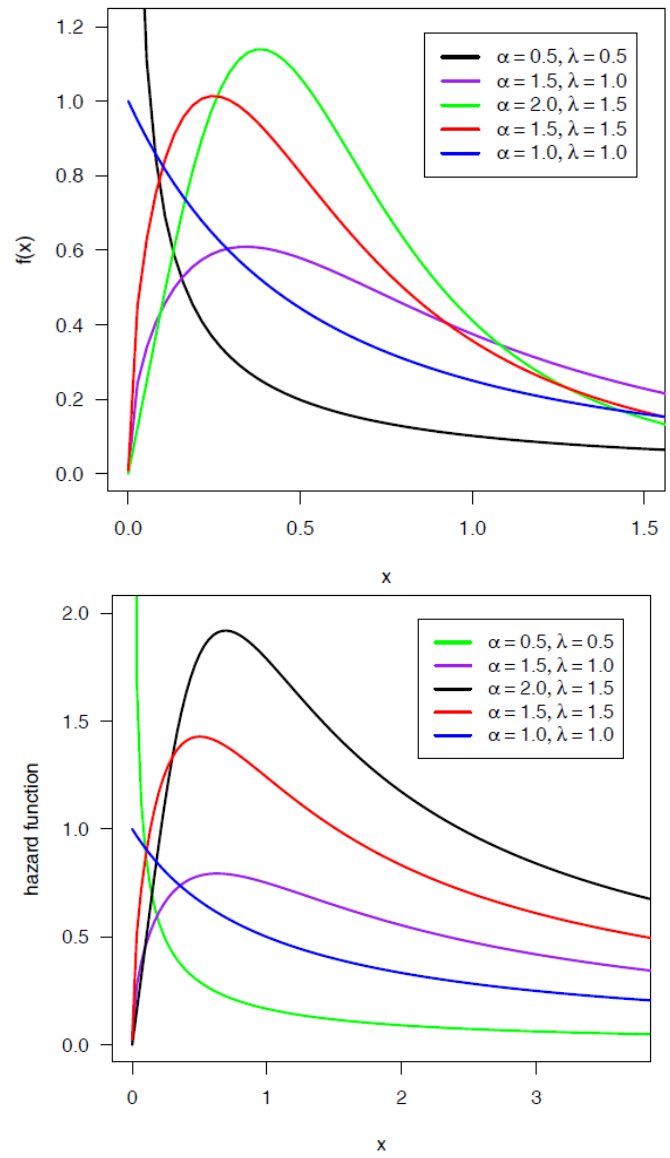

Figure 1. Plots of PDF (upper panel) and hazard function (lower panel)

The Quantile function of Logistic Lomax distribution is

$$
x_{p}=\frac{1}{\beta}\left[\left\{\left(\frac{p}{1-p}\right)^{1 / \alpha}+1\right\}^{1 / \lambda}-1\right] ; 0<p<1 \text {. }
$$

Random deviate generation:

$$
=\frac{1}{\beta}\left[\left\{1+\left(\frac{1}{u}-1\right)^{-1 / \alpha}\right\}^{1 / \lambda}-1\right] ; 0<\mathrm{u}<1
$$

Skewness and Kurtosis:

The measures of Skewness based on quantiles is Bowley's coefficient of skewness and it can be expressed as

$$
\text { Skewness }=\frac{Q(0.75)+Q(0.25)-2 Q(0.5)}{Q(0.75)-Q(0.25)}
$$

Coefficient of kurtosis based on octiles which was defined by (Moors, 1988) is

$$
K_{u}(M)=\frac{Q(0.875)-Q(0.625)+Q(0.375)-Q(0.125)}{Q(3 / 4)-Q(1 / 4)},
$$




\section{Methods of estimation}

For the parameter estimates of the presented distribution, MLE estimates is used along with CVME and LSE methods in this section.

\subsection{Maximum Likelihood Estimates}

Consider, $x_{1}, x_{2}, \ldots, x_{n}$ is random sample from $L L(\alpha, \beta, \lambda)$ and the likelihood function, $L(\alpha, \beta, \lambda)$ is given by,

$$
\begin{aligned}
& L\left(\psi ; x_{1}, x_{2} \ldots x_{n}\right)=f\left(x_{1}, x_{2}, \ldots x_{n} / \psi\right)=\prod_{i=1}^{n} f\left(x_{i} / \psi\right) \\
& L(\alpha, \beta, \lambda)=\alpha \beta \lambda \prod_{i=1}^{n} \frac{\left(1+\beta x_{i}\right)^{\lambda-1}\left(\left(1+\beta x_{i}\right)^{\lambda}-1\right)^{\alpha-1}}{\left\{1+\left(\left(1+\beta x_{i}\right)^{\lambda}-1\right)^{\alpha}\right\}^{2}}
\end{aligned}
$$

$; x \geq 0 ;(\alpha, \beta, \lambda)>0, x>0$

Now log-likelihood density is

$$
\begin{aligned}
& l=n \ln \alpha+n \ln \beta+n \ln \lambda+(\lambda-1) \sum_{i=1}^{n} \log \left(1+\beta x_{i}\right) \\
& +(\alpha-1) \sum_{i=1}^{n} \log \left\{\left(1+\beta x_{i}\right)^{\lambda}-1\right\}-2 \sum_{i=1}^{n} \log \left[1+\left\{\left(1+\beta x_{i}\right)^{\lambda}-1\right\}^{\alpha}\right]
\end{aligned}
$$

Differentiation of (3.1.1) with respect to $\alpha, \beta$ and $\lambda$ we get,

$$
\frac{\partial l}{\partial \alpha}=\frac{n}{\alpha}+\sum_{i=1}^{n} \log \left\{\left(1+\beta x_{i}\right)^{\lambda}-1\right\}-2 \sum_{i=1}^{n} \frac{\left\{\left(1+\beta x_{i}\right)^{\lambda}-1\right\}^{\alpha} \log \left\{\left(1+\beta x_{i}\right)^{\lambda}-1\right\}}{\left.1+\left(1+\beta x_{i}\right)^{\lambda}-1\right\}^{\alpha}}
$$$$
\frac{\partial l}{\partial \beta}=\frac{n}{\beta}+(\lambda-1) \sum_{i=1}^{n} \frac{x_{i}}{1+\beta x_{i}}+\lambda(\alpha-1) \sum_{i=1}^{n} \frac{x_{i}\left(1+\beta x_{i}\right)^{\lambda-1}}{\left(1+\beta x_{i}\right)^{\lambda-1}-1}
$$$$
-2 \alpha \lambda \sum_{i=1}^{n} \frac{x_{i}\left(1+\beta x_{i}\right)^{\lambda-1}\left\{\left(1+\beta x_{i}\right)^{\lambda}-1\right\}^{\alpha-1}}{1+\left\{\left(1+\beta x_{i}\right)^{\lambda}-1\right\}}
$$$$
\frac{\partial l}{\partial \lambda}=\frac{n}{\lambda}+\sum_{i=1}^{n} \log \left(1+\beta x_{i}\right)+(\alpha-1) \sum_{i=1}^{n} \frac{\left(1+\beta x_{i}\right)^{\lambda} \log \left(1+\beta x_{i}\right)}{\left(1+\beta x_{i}\right)^{\lambda}-1}
$$

$$
-2 \alpha \sum_{i=1}^{n} \frac{\left\{\left(1+\beta x_{i}\right)^{\lambda}-1\right\}^{\alpha-1}\left(1+\beta x_{i}\right)^{\lambda} \log \left(1+\beta x_{i}\right) x_{i}}{1+\left\{\left(1+\beta x_{i}\right)^{\lambda}-1\right\}^{\alpha}}
$$

We get the ML estimates $\hat{\alpha}, \hat{\beta}$ and $\hat{\lambda}$, equating above equations to zero and solving for the unknown parameters i.e. $\alpha, \beta$ and $\lambda$, Maximizing (3.1.1) with software platforms like $\mathrm{R}$, we can get the unknown parameters' estimated values. For the confidence interval estimation of $\alpha, \beta$ and $\lambda$ and testing of the hypothesis, we have to calculate the observed information matrix. The observed information matrix of $\alpha, \beta$ and $\lambda$ for the confidence interval estimation and testing the hypothesis is given as,

$U=\left[\begin{array}{lll}U_{11} & U_{12} & U_{13} \\ U_{21} & U_{22} & U_{23} \\ U_{31} & U_{32} & U_{33}\end{array}\right]$

Where

$U_{11}=\frac{\partial^{2} l}{\partial \alpha^{2}}, U_{12}=\frac{\partial^{2} l}{\partial \alpha \partial \beta}, U_{13}=\frac{\partial^{2} l}{\partial \alpha \lambda}$

$$
\begin{aligned}
& U_{21}=\frac{\partial^{2} l}{\partial \beta \partial \alpha}, U_{22}=\frac{\partial^{2} l}{\partial \beta^{2}}, U_{23}=\frac{\partial^{2} l}{\partial \beta \partial \lambda} \\
& U_{31}=\frac{\partial^{2} l}{\partial \lambda \partial \alpha}, U_{32}=\frac{\partial^{2} l}{\partial \beta \partial \lambda}, U_{33}=\frac{\partial^{2} l}{\partial \lambda^{2}}
\end{aligned}
$$

Let $\Omega=(\alpha, \beta, \lambda)$ denote the parameter space and the corresponding MLE of $\Omega$ as $\hat{\Omega}=(\hat{\alpha}, \hat{\beta}, \hat{\lambda})$, then $(\hat{\Omega}-\Omega) \rightarrow N_{3}\left[0,(U(\Omega))^{-1}\right]$ where $U(\Omega)$ is the information matrix of Fisher. With help of Newton-Raphson method maximizing likelihood gives the observed information matrix and hence the variance-covariance matrix is obtained as,

$$
[U(\Omega)]^{-1}=\left(\begin{array}{ccc}
\operatorname{var}(\hat{\alpha}) & \operatorname{cov}(\hat{\alpha}, \hat{\beta}) & \operatorname{cov}(\hat{\alpha}, \hat{\lambda}) \\
\operatorname{cov}(\hat{\alpha}, \hat{\beta}) & \operatorname{var}(\hat{\beta}) & \operatorname{cov}(\hat{\beta}, \hat{\lambda}) \\
\operatorname{cov}(\hat{\alpha}, \hat{\lambda}) & \operatorname{cov}(\hat{\beta}, \hat{\lambda}) & \operatorname{var}(\hat{\lambda})
\end{array}\right)
$$

Thus approximate $100(1-\alpha) \%$ confidence intervals for $\alpha, \beta$ and $\lambda$ from the asymptotic normality of MLEs can be constructed as,

$\hat{\beta} \pm Z_{\alpha / 2} S E(\hat{\beta}), \hat{\lambda} \pm Z_{\alpha / 2} S E(\hat{\lambda})$ and, $\hat{\alpha} \pm Z_{\alpha / 2} S E(\hat{\alpha})$

Here upper percentile of standard normal variate is denoted by $Z_{\alpha / 2}$

\subsection{Least-Square Estimation (LSE) Method}

The least-square estimators (Swain et al, 1988) of the unknown parameters $\alpha, \beta$ and $\lambda$ of LL distribution can be calculated with minimization of the following equation with respect to unknown parameters $\alpha, \beta$ and $\lambda$.

$M(X ; \alpha, \beta, \lambda)=\sum_{i=1}^{n}\left[F\left(X_{i}\right)-\frac{i}{n+1}\right]^{2}$

Let $F\left(X_{i}\right)$ represent ordered random variables' distribution function $\mathrm{X}_{(1)}<\mathrm{X}_{(2)}<\ldots<\mathrm{X}_{(\mathrm{n})}$ here $\left\{X_{1}, X_{2}, \ldots, X_{n}\right\}$ is a random sample of size $\mathrm{n}$ from a distribution function $\mathrm{F}($.). The least-square estimators $\hat{\alpha}, \hat{\beta}$, and $\hat{\lambda}$ of $\alpha, \beta$ and $\lambda$ is acquired with minimization of the following equation with respect to $\alpha, \beta$ and $\lambda$.

$M(X ; \alpha, \beta, \lambda)=\sum_{i=1}^{n}\left[1-\frac{1}{1+\left\{\left(1+\beta x_{i}\right)^{\lambda}-1\right\}^{\alpha}}-\frac{i}{n+1}\right]^{2}$

; $x \geq 0,(\alpha, \beta, \lambda)>0$

Differentiation of (3.2.2) with respect to $\alpha, \beta$ and $\lambda$ we obtain,

$$
\frac{\partial M}{\partial \alpha}=-2 \sum_{i=1}^{n}\left[1-\frac{1}{1+\left[B\left(x_{i}\right)\right]^{\alpha}}-\frac{i}{n+1}\right] \frac{\left[B\left(x_{i}\right)\right]^{\alpha} \ln \left[B\left(x_{i}\right)\right]}{\left\{1+\left[B\left(x_{i}\right)\right]^{\alpha}\right\}^{2}}
$$


$\frac{\partial M}{\partial \beta}=-2 \alpha \lambda \sum_{i=1}^{n} x_{i}\left[1-\frac{1}{1+\left[B\left(x_{i}\right)\right]^{\alpha}}-\frac{i}{n+1}\right] \frac{\left[B\left(x_{i}\right)\right]^{\alpha-1}\left(1+\beta x_{i}\right)^{\lambda-1}}{\left\{1+\left[B\left(x_{i}\right)\right]^{\alpha}\right\}^{2}}$

$\frac{\partial M}{\partial \lambda}=-2 \lambda \sum_{i=1}^{n}\left[1-\frac{1}{1+\left[B\left(x_{i}\right)\right]^{\alpha}}-\frac{i}{n+1}\right] \frac{\left[B\left(x_{i}\right)\right]^{\alpha-1}\left(1+\beta x_{i}\right)^{\lambda-1}}{\left\{1+\left[B\left(x_{i}\right)\right]^{\alpha}\right\}^{2}}$

Where $B\left(x_{i}\right)=\left(1+\beta x_{i}\right)^{\lambda}-1$

Likewise we obtain weighted least square estimators with the minimization of

$M(X ; \alpha, \beta, \lambda)=\sum_{i=1}^{n} w_{i}\left[F\left(X_{(i)}\right)-\frac{i}{n+1}\right]^{2}$

with respect to $\alpha, \beta$ and $\lambda$. The weights $w_{i}$ are $w_{i}=\frac{1}{\operatorname{Var}\left(X_{(i)}\right)}=\frac{(n+1)^{2}(n+2)}{i(n-i+1)}$

Thus we can get weighted least square estimators of $\alpha, \beta$ and $\lambda$ with respect to $\alpha, \beta$ and $\lambda$.

$M(X ; \alpha, \beta, \lambda)=$

$\sum_{i=1}^{n} \frac{(n+1)^{2}(n+2)}{i(n-i+1)}\left[1-\frac{1}{1+\left\{\left(1+\beta x_{i}\right)^{\lambda}-1\right\}^{\alpha}}-\frac{i}{n+1}\right]^{2}$

\subsection{Method of Cramer-Von-Mises estimation (CVME)}

The Cramer-Von-Mises estimators of $\alpha, \beta$ and $\lambda$ are acquired with minimization of the following equation

$$
\begin{aligned}
& C(X ; \alpha, \beta, \lambda)=\frac{1}{12 n}+\sum_{i=1}^{n}\left[F\left(x_{i: n} \mid \alpha, \beta, \lambda\right)-\frac{2 i-1}{2 n}\right]^{2} \\
& =\frac{1}{12 n}+\sum_{i=1}^{n}\left[1-\frac{1}{1+\left\{\left(1+\beta x_{i}\right)^{\lambda}-1\right\}^{\alpha}}-\frac{2 i-1}{2 n}\right]^{2}
\end{aligned}
$$

Differentiation of (3.3.1) with respect to $\alpha, \beta$ and $\lambda$ we get,

$$
\frac{\partial C}{\partial \alpha}=-2 \sum_{i=1}^{n}\left[1-\frac{1}{1+\left[B\left(x_{i}\right)\right]^{\alpha}}-\frac{2 i-1}{2 n}\right] \frac{\left[B\left(x_{i}\right)\right]^{\alpha} \ln \left[B\left(x_{i}\right)\right]}{\left\{1+\left[B\left(x_{i}\right)\right]^{\alpha}\right\}^{2}}
$$

$\frac{\partial C}{\partial \beta}=$

$-2 \alpha \lambda \sum_{i=1}^{n} x_{i}\left[1-\frac{1}{1+\left[B\left(x_{i}\right)\right]^{\alpha}}-\frac{2 i-1}{2 n}\right] \frac{\left[B\left(x_{i}\right)\right]^{\alpha-1}\left(1+\beta x_{i}\right)^{\lambda-1}}{\left\{1+\left[B\left(x_{i}\right)\right]^{\alpha}\right\}^{2}}$

$\frac{\partial C}{\partial \lambda}=-2 \lambda \sum_{i=1}^{n}\left[1-\frac{1}{1+\left[B\left(x_{i}\right)\right]^{\alpha}}-\frac{2 i-1}{2 n}\right] \frac{\left[B\left(x_{i}\right)\right]^{\alpha-1}\left(1+\beta x_{i}\right)^{\lambda-1}}{\left\{1+\left[B\left(x_{i}\right)\right]^{\alpha}\right\}^{2}}$
Where $B\left(x_{i}\right)=\left(1+\beta x_{i}\right)^{\lambda}-1$

We obtain CVM estimators solving $\frac{\partial C}{\partial \alpha}=0, \frac{\partial C}{\partial \beta}=0$ and $\frac{\partial C}{\partial \lambda}=0$ simultaneously.

\section{REAL DATASET APPLICATIONS}

Here, we demonstrate the real life application of logistic Lomax distribution using a real dataset. This is a real data set represents the remission times (in months) of a random sample of 128 bladder cancer patients (Lee \& Wang, 2003): sorted data

$0.08,0.20,0.40,0.50,0.51,0.81,0.90,1.05,1.19,1.26,1.35$ $1.40,1.46,1.76,2.02,2.02,2.07,2.09,2.23,2.26,2.46,2.54$ $2.62,2.64,2.69,2.69,2.75,2.83,2.87,3.02,3.25,3.31,3.36$, $3.36,3.48,3.52,3.57,3.64,3.70,3.82,3.88,4.18,4.23,4.26$, $4.33,4.34,4.40,4.50,4.51,4.87,4.98,5.06,5.09,5.17,5.32$, $5.32,5.34,5.41,5.41,5.49,5.62,5.71,5.85,6.25,6.54,6.76$ $6.93,6.94,6.97,7.09,7.26,7.28,7.32,7.39,7.59,7.62,7.63$, $7.66,7.87,7.93,8.26,8.37,8.53,8.65,8.66,9.02,9.22,9.47$, $9.74,10.06,10.34,10.66,10.75,11.25,11.64,11.79,11.98$, $12.02,12.03,12.07,12.63,13.11,13.29,13.80,14.24,14.76$, $14.77,14.83,15.96,16.62,17.12,17.14,17.36,18.10,19.13$ $20.28,21.73,22.69,23.63,25.74,25.82,26.31,32.15,34.26$, $36.66,43.01,46.12,79.05$

The MLEs are calculated with the help of the optim() function in R platform (R Core Team, 2020) and (Mailund, 2017) with minimization of the likelihood function (3.1.1). We have obtained Log-Likelihood value is $l=-409.6158$ and the MLE's with their standard errors (SE) for $\alpha, \beta$, and $\lambda$ are presented in Table 1.

Table 1

MLE and SE for $\alpha, \beta$ and $\lambda$

\begin{tabular}{ccc} 
Parameter & MLE & \multicolumn{1}{c}{ SE } \\
\hline alpha & 1.38027 & 0.17020 \\
beta & 0.04451 & 0.03440 \\
lambda & 2.80412 & $1.849531 .4 .1)$
\end{tabular}

We have demonstrated the plots of the profile log-likelihood function in Figure 2 (Kumar \& Ligges, 2011) where we can see the MLEs are unique.
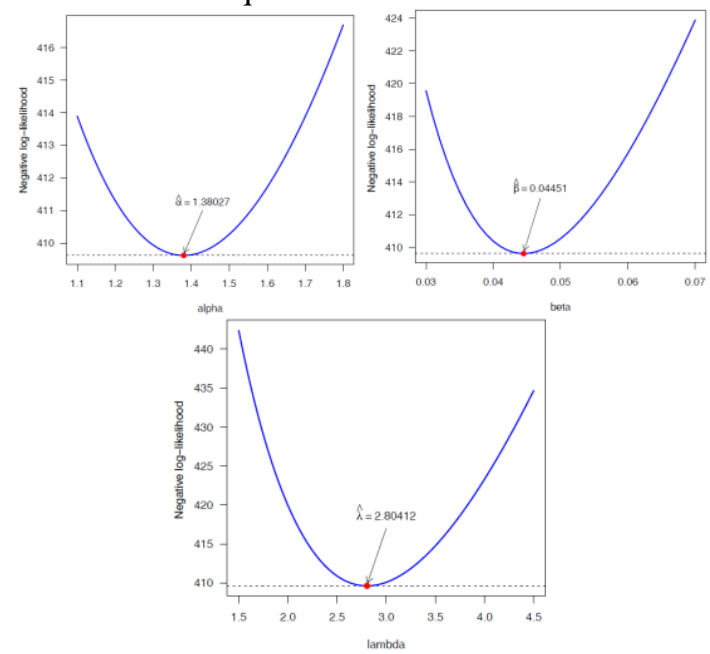
Figure 2. Graph of profile $\log$-likelihood function of $\alpha, \beta$, and $\lambda$.

In Figure 3 we illustrate the Q-Q plot and P-P plot of LL distribution.
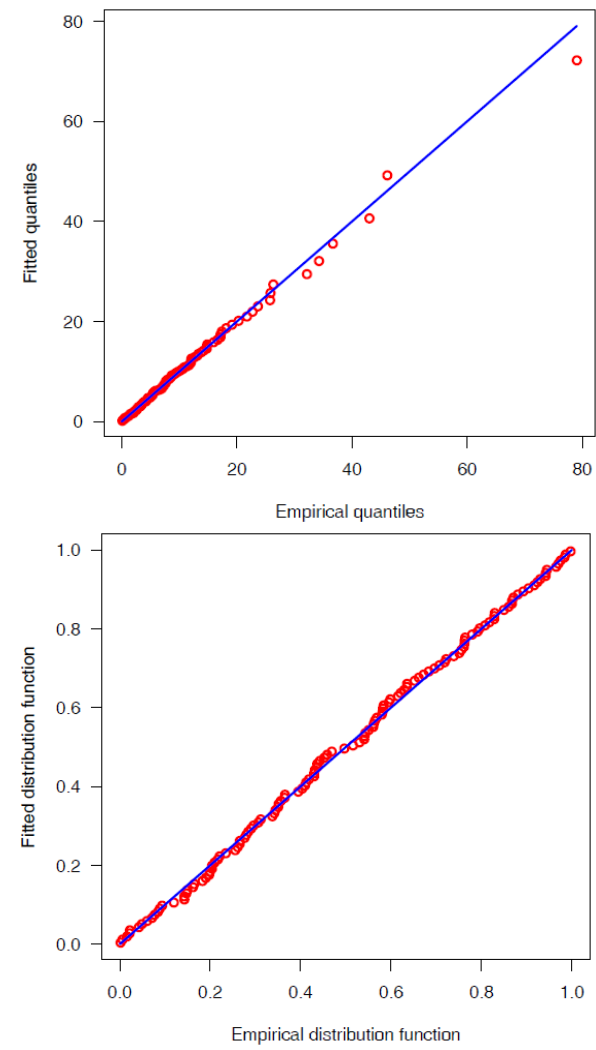

Figure 3. The Q-Q plot (upper panel) and P-P plot (lower panel) of LL distribution

In Table 2 we illustrate estimated value of the parameters of LL distribution using MLE, LSE and CVE method and their corresponding negative log-likelihood, AIC and KS criterion.

Table 2

Estimated parameters, log-likelihood, and AIC

\begin{tabular}{cccc}
\hline $\begin{array}{c}\text { Method of } \\
\text { Estimation }\end{array}$ & MLE & LSE & CVE \\
\hline$\hat{\alpha}$ & 1.38027 & 1.49883 & 1.51267 \\
\hline$\hat{\beta}$ & 0.04451 & 0.07583 & 0.07443 \\
\hline$\hat{\lambda}$ & 2.80412 & 1.79072 & 1.81832 \\
\hline -LL & 409.6158 & 409.9209 & 409.9223 \\
\hline AIC & 825.2317 & 825.8418 & 825.8446 \\
\hline KS(p-value) & $0.0335(0.9988)$ & $0.0301(0.9998)$ & $0.0306(0.9998)$ \\
\hline
\end{tabular}

In Figure 4, we have plotted the histogram and the density function of fitted distributions and Q-Q plot of estimation methods MLE, LSE and CVM.
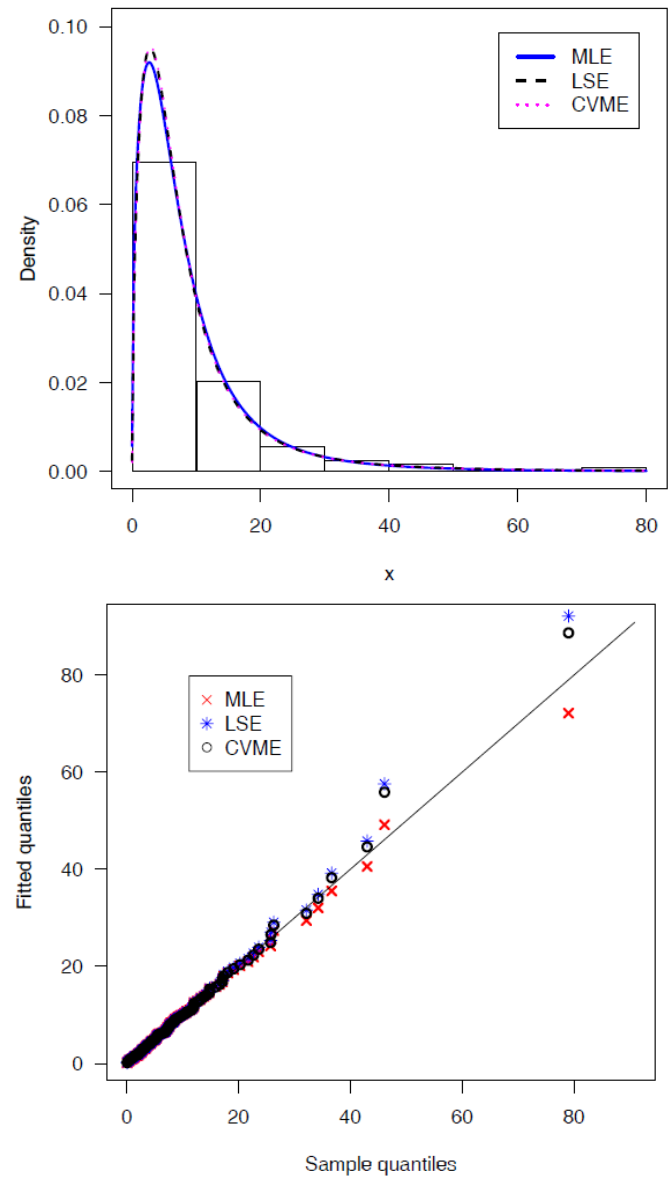

Figure 4. The Histogram and the density function of fitted distributions of estimation methods MLE, LSE and CVM (upper panel) and fitted quantiles and sample quantiles (lower panel) of LL distribution.

Following distributions are used for comparison purpose of goodness of fit.

A. Exponentiated Exponent4al Poisson (EEP):

The PDF of EEP (Ristić \& Nadarajah, 2014) can be expressed as

$f(x)=\frac{\alpha \beta \lambda}{\left(1-e^{-\lambda}\right)} e^{-\beta x}\left(1-e^{-\beta x}\right)^{\alpha-1} \exp \left\{-\lambda\left(1-e^{-\beta x}\right)^{\alpha}\right\}$ $; x>0, \alpha>0, \lambda>0$

B. Exponentiated Lomax (EL) distribution:

The CDF of exponentiated lomax introduced by (Lan \& Leemis, 2008) is

$$
\begin{aligned}
& F(x)=\left\{1-(1+\beta x)^{-\alpha}\right\}^{\lambda} \\
& ; x \geq 0, \alpha>0, \beta>0, \lambda>0 .
\end{aligned}
$$

C. Generalized Exponential Extension (GEE) DISTRIBUTION:

The PDF of GEE introduced by (Lemonte, 2013) with parameters $\alpha, \beta$ and $\lambda$ is

$$
\begin{array}{r}
f_{G E E}(x ; \alpha, \beta, \lambda)=\alpha \beta \lambda(1+\lambda x)^{\alpha-1} \exp \left\{1-(1+\lambda x)^{\alpha}\right\} \\
{\left[1-\exp \left\{1-(1+\lambda x)^{\alpha}\right\}\right]^{\beta-1} ; x \geq 0 .}
\end{array}
$$




\section{Generalized Exponential (GE) distribution:}

The PDF of generalized exponential distribution (Gupta \& Kundu, 1999) is.

$$
\begin{aligned}
& f_{G E}(x ; \alpha, \lambda)=\alpha \lambda e^{-\lambda x}\left\{1-e^{-\lambda x}\right\}^{\alpha-1} \\
& ;(\alpha, \lambda)>0, x>0
\end{aligned}
$$

E. Exponential power (EP) distribution:

The PDF of Exponential power (EP) distribution (Smith \& Bain, 1975) is

$$
f_{E P}(x)=\alpha \lambda^{\alpha} x^{\alpha-1} e^{(\lambda x)^{\alpha}} \exp \left\{1-e^{(\lambda x)^{\alpha}}\right\}
$$$$
;(\alpha, \lambda)>0, \quad x \geq 0
$$

where $\alpha$ and $\lambda$ are the shape and scale parameters, respectively.

For the assessment of potentiality of the proposed model we have meaured the Akaike information criterion (AIC), Bayesian information criterion (BIC), Corrected Akaike information criterion (CAIC) and Hannan-Quinn information criterion (HQIC) which are presented in Table 3.

Table 3

Log-likelihood (LL), AIC, BIC, CAIC and HQIC

\begin{tabular}{lccccc}
\hline $\begin{array}{l}\text { Distri } \\
\text { butio } \\
\text { n }\end{array}$ & -LL & AIC & BIC & CAIC & HQIC \\
\hline LL & 409.6158 & 825.2317 & 833.7877 & 825.4252 & 828.7080 \\
EEP & 409.7528 & 825.5056 & 834.0617 & 825.6991 & 828.9819 \\
EL & 410.0718 & 826.1436 & 834.6997 & 826.3372 & 829.6200 \\
GEE & 410.6013 & 827.2026 & 835.7586 & 827.3961 & 830.6789 \\
GE & 413.0776 & 830.1552 & 835.8592 & 830.2512 & 832.4728 \\
EP & 426.6474 & 857.2948 & 862.9989 & 857.3893 & 859.6124 \\
\hline
\end{tabular}

Figure 4 illustrates the comparison made between the distributions.
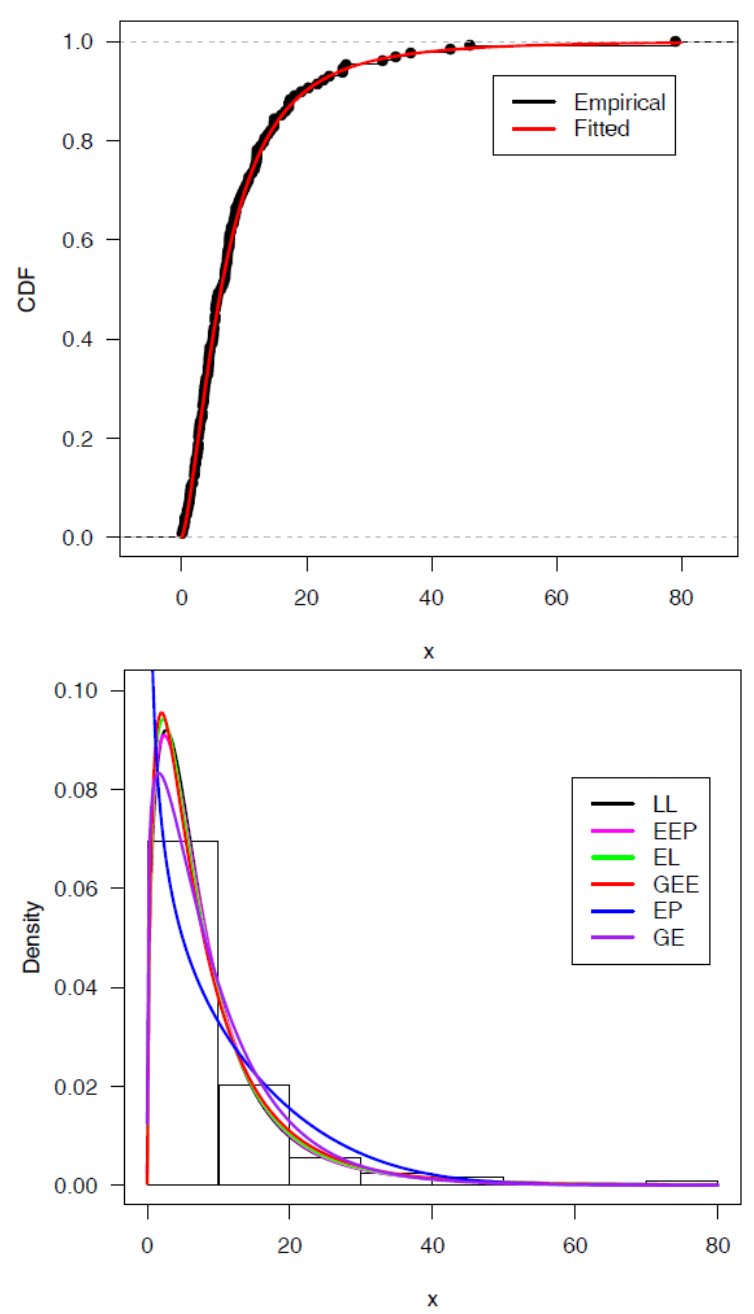

Figure 5. Empirical distribution function with estimated distribution function (upper panel) and The Histogram and the density function of fitted distributions (lower panel)

Different p-values in the Cramer-Von Mises $\left(\mathrm{A}^{2}\right)$, the Anderson-Darling (W) and Kolmogorov-Simnorov (KS) statistics are presented in Table 4 for comparing the goodness-of-fit of the LL distribution with other competing distributions. With minimum value of test statistic and higher p-value, we can observe that in comparison to the distributions taken, LL distribution gives better fit with more consistency and reliability in results.

\section{Table 4}

The goodness-of-fit statistics and their corresponding $\mathrm{p}$-value

\begin{tabular}{lccc}
\hline Distribution & $\boldsymbol{K S}(\boldsymbol{p}$-value $)$ & $\boldsymbol{W}(\boldsymbol{p}$-value $)$ & $\boldsymbol{A}^{2}(\boldsymbol{p}$-value $)$ \\
\hline LL & $0.0335(0.9988)$ & $0.0161(0.9994)$ & $0.1115(0.9999)$ \\
EEP & $0.0380(0.9925)$ & $0.0220(0.9946)$ & $0.1486(0.9987)$ \\
EL & $0.0405(0.9846)$ & $0.0262(0.9871)$ & $0.1798(0.9950)$ \\
GEE & $0.0442(0.9636)$ & $0.0394(0.9367)$ & $0.2630(0.9631)$ \\
GE & $0.0725(0.5115)$ & $0.1279(0.4652)$ & $0.7137(0.5472)$ \\
EP & $0.1199(0.0503)$ & $0.5993(0.0223)$ & $3.6745(0.0126)$ \\
\hline
\end{tabular}




\section{CONCLUSION}

In the presented work, a continuous distribution with three-parameter called Logistic Lomax distribution is presented along with relevant distributional properties like PDF, CDF, hazard rate function, quantile function to build a better understanding of the distribution introduced. We have found that the model is flexible with inverted bathtub shaped hazard function. For the parameter estimates of the presented distribution, Maximum likelihood Estimation (MLE) is used along with Cramer-Von-Mises estimation (CVME) and least-square estimation (LSE) methods where MLE gave better outcome. On analysis of a real data set, we can observe that with minimum value of test statistic and higher p-value, in comparison to the distributions taken, LL distribution gives better fit with more consistency and reliability results. With such observation, we hope that in the field of survival analysis the proposed distribution can be an alternative to the existing distributions.

\section{REFERENCES}

[1] Chaudhary, A. K. \& Kumar, V. (2020). Half logistic exponential extension distribution with Properties and Applications. International Journal of Recent Technology and Engineering (IJRTE), 8(3), 506-512.

[2] Chaudhary, A. K. \& Kumar, V. (2020). Lindley half Cauchy distribution: Properties and Applications. International Journal for Research in Applied Science \& Engineering Technology (IJRASET), 8(9), 1233-1242.

[3] Hassan, A. and Al-Ghamdi, A. (2009). Optimum step stress accelerated life testing for Lomax distribution. Journal of Applied Sciences Research, 5, 2153-2164.

[4] Joshi, R. K. \& Kumar, V. (2020). Half Logistic NHE: Properties and Application. International Journal for Research in Applied Science \& Engineering Technology (IJRASET), 8(9), 742-753.

[5] Joshi, R. K., Sapkota, L.P. \& Kumar, V. (2020). The Logistic-Exponential Power Distribution with Statistical Properties and Applications, International Journal of Emerging Technologies and Innovative Research, 7(12), 629-641

[6] Kumar, V. (2010). Bayesian analysis of exponential extension model. J. Nat. Acad. Math, 24, 109-128.

[7] Kumar, V. and Ligges, U. (2011). reliaR: A package for some probability distributions http://cran.r-project.org/web/packages/reliaR/index.html.

[8] Lan, Y., \& Leemis, L. M. (2008). The logistic-exponential survival distribution. Naval Research Logistics (NRL), 55(3), 252-264.

[9] Lee, E. T. \& Wang, J. (2003). Statistical methods for survival data analysis (Vol. 476). John Wiley \& Sons.

[10] Lemonte, A. J. (2013). A new exponential-type distribution with constant, decreasing, increasing, upside-down bathtub and bathtub-shaped failure rate function. Computational Statistics \& Data Analysis, 62, 149-170.

[11] Lomax, K. S. (1954). Business failures: Another example of the analysis of failure data. Journal of the American Statistical Association, 49(268), 847-852.

[12] Mailund, T. (2017). Functional Programming in R: Advanced Statistical Programming for Data Science, Analysis and Finance. Apress, Aarhus N, Denmark ISBN-13 (pbk): 978-1-4842-2745-9 ISBN-13 (electronic): 978-1-4842-2746-6 DOI 10.1007/978-1-4842-2746-6

[13] Mandouh, R. M. (2018). Logistic-modified weibull distribution and parameter estimation. International Journal of Contemporary Mathematical Sciences, 13(1), 11-23.

[14] Moors, J. (1988). A quantile alternative for kurtosis. The Statistician, $37,25-32$.

[15] R Core Team (2020). R: A language and environment for statistical co mputing. R Foundation for Statistical Computing, Vienna, Austria. UR L https://www.R-project.org/.

[16] Ristić, M. M., \& Nadarajah, S. (2014). A new lifetime distribution. Journal of Statistical Computation and Simulation, 84(1), 135-150.

[17] Smith, R.M. and Bain, L.J. (1975). An exponential power life-test distribution, Communications in Statistics, 4, 469-481
[18] Swain, J. J., Venkatraman, S. \& Wilson, J. R. (1988). Least-squares estimation of distribution functions in johnson's translation system. Journal of Statistical Computation and Simulation, 29(4), 271-297.

[19] Tahir, M. H., Cordeiro, G. M., Alzaatreh, A., Mansoor, M., \& Zubair, M. (2016). The logistic-X family of distributions and its applications. Communications in Statistics-Theory and Methods, 45(24), 7326-7349.

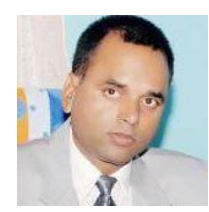

Arun Kumar Chaudhary received his M.Sc in Statistics from Central Department of Statistics, Tribhuwan University and $\mathrm{PhD}$ in Statistics from D.D.U. Gorakhpur University, India. Currently working as Associate Professor in Department of Management Science(Statistics), Nepal Commerce Campus, Tribhuwan University, Nepal. He has got 26 years of teaching experience. He is associated with many other reputed colleges as visiting faculty. He is a life member of Nepal Statistical Association (NEPSA) and vice-chairperson of Nepal Statistical Society (NESS). He has authored more than 30 textbooks on statistics and mathematics.

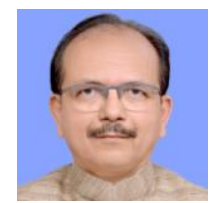

Vijay Kumar received his M.Sc and Ph.D. in Statistics from D.D.U. Gorakhpur University. Currently working as Professor in Department of Mathematics and Statistics in DDU Gorakhpur University, Gorakhpur U.P. He has got 27 years of teaching/research experience. $\mathrm{He}$ is visiting Faculty of MaxPlanck-Institute, Germany. His main research interests are Reliability Engineering, Bayesian Statistics, and Actuarial Science. $9 \mathrm{PhD}$ scholars have been awarded under his supervision and currently he is supervising $4 \mathrm{PhD}$ scholars. He has published more than 40 national and international articles. 